\title{
ANNOUNCEMENT
}

\section{Change in Editorship}

Patricia Morgan Meyer will assume editorship of Physiological Psychology in 1982. After June 30, 1981, please send manuscripts to her at: Ohio State University Research Center, 1314 Kinnear Road, Columbus, Ohio 43212. 